\title{
Horizons/Théâtre
}

Revue d'études théâtrales

12 | 2018

Les dramaturgies arabes et l'Occident

\section{Transfert des esthétiques politiques du théâtre européen dans le contexte égyptien chez Laila Soliman et Ahmed el-Attar}

Pauline Donizeau

\section{(2) OpenEdition \\ 1 Journals}

Édition électronique

URL : http://journals.openedition.org/ht/358

DOI : $10.4000 /$ ht.358

ISSN : 2678-5420

Éditeur

Presses universitaires de Bordeaux

\section{Édition imprimée}

Date de publication : 1 janvier 2018

Pagination : 84-96

ISSN : 2261-4591

\section{Référence électronique}

Pauline Donizeau, «Transfert des esthétiques politiques du théâtre européen dans le contexte égyptien chez Laila Soliman et Ahmed el-Attar », Horizons/Théâtre [En ligne], 12 | 2018, mis en ligne le 01 janvier 2019, consulté le 21 juillet 2019. URL : http://journals.openedition.org/ht/358 ; DOI :

10.4000/ht.358

\section{(c) (i) $\odot$}

La revue Horizons/Théâtre est mise à disposition selon les termes de la Licence Creative Commons Attribution - Pas d'Utilisation Commerciale - Pas de Modification 4.0 International. 


\section{Pauline Donizeau}

Pauline Donizeau, diplômée de l'École Normale Supérieure de Paris et agrégée d'Histoire, est actuellement doctorante contractuelle en Études théâtrales à l'Université Paris Nanterre, et chercheuse associée au CEDEJ (centre d'études et de documentation économique, juridique et sociale) basé au Caire. Elle prépare une thèse sous la direction de Christian Biet. Ses recherches portent sur la notion de théâtre politique, à travers l'étude du théâtre égyptien au cours de la période révolutionnaire et sa genèse (2004-2017). Elle s'intéresse également à l'histoire culturelle de l'Égypte et aux circulations culturelles à l'échelle du Moyen-Orient (Égypte-Syrie-Liban) et entre Orient et Occident. Elle enseigne l'Histoire du théâtre et la dramaturgie en à l'Université ParisNanterre. Parallèlement à ses activités de recherche, elle est également conseillère scientifique et dramaturgique au sein de la compagnie de théâtre " Les Temps blancs ».

Mail: p.donizeau@hotmail.fr

Résumé: Dans le contexte d'échanges culturels entre l'Égypte et l'Occident depuis le $x \mid x^{e}$ siècle, la génération du théâtre indépendant qui a cherché, à partir des années 1990, à créer un nouveau théâtre engagé dans les problématiques sociales et politiques, et a été largement influencée par les esthétiques occidentales et les formes du théâtre politique. Ahmed el-Attar, qui emprunte les formes du théâtre épique brechtien dans son spectacle Mother I want to be a millionnaire (2004) et Laila Soliman, proposant un théâtre documentaire dans son spectacle No Time For Art (2011), en sont des exemples pertinents. Si l'étude de leurs dramaturgies nous permet d'étudier les dynamiques de transferts culturels de l'Occident vers l'Orient, cet article propose également de voir en quoi, à l'inverse, ces spectacles sont conçus pour un double public, à la fois occidental et égyptien, et sont donc pensés comme des objets transculturels, en interrogeant ce double horizon de création et de réception.

Mots-cLÉs: Transferts culturels, Égypte, esthétique politique, Brecht, théâtre épique, théâtre documentaire
Abstract: In the context of cultural exchanges between Egypt and the West since the 19th century, the new generation of the independent theatre which tried, from the nineties, to produce a new theatre, politically and socially engaged, has been influenced by western aesthetics and forms of political theatre. Ahmed el-Attar, who use elements of Brecht's epic theatre in his play Mother I want to be a millionaire (2004), and Laila Soliman, who makes documentary theatre in the performance No Time For Art (2011) are good examples. If we can study the dynamics of cultural transfers from the West to the East through their works, this article also tackles the issue of the transfer from the East to the West, as these performances are conceived for a double audience, both European and Egyptian, and have to be considered as transcultural objects.

Kerwords: Cultural transfers, Egypt, political aesthetics, Brecht, epic theatre, documentary theatre 


\section{Transfert des esthétiques politiques du théâtre européen dans le contexte égyptien chez Laila Soliman et Ahmed el-Attar}

LAILA Soliman ET AHMEd EL-ATTAR sont aujourd' hui des artistes majeurs de la scène théâtrale égyptienne, à la fois en Égypte, mais aussi en Occident, grâce à leur visibilité sur les scènes européennes. Ils font partie d'un ensemble d'artistes qui ont donné ces dernières années à la scène égyptienne une vivacité nouvelle, devenue d'autant plus visible dans notre prisme occidental depuis la révolution de 2011. Ces deux dramaturges proposent un théâtre que l'on pourrait faire entrer dans la catégorie du «théâtre politique », même si cette notion demeure floue, dans la mesure où sa définition ne cesse de se transformer au gré des situations dans lesquelles un tel théâtre est produit. On pourrait le définir, dans le cas de ces deux artistes, comme « un théâtre motivé par "quelque chose" qui l'excède ou le justifie ${ }^{1} \gg$, ou comme un théâtre cherchant à avoir « une capacité mobilisatrice et transformatrice ${ }^{2}$ », d'après des définitions proposées par Olivier Neveux dans son ouvrage Politiques du spectateur. Ce théâtre cherche en effet à interroger la société, et aborde à la scène des thèmes politiques. Le caractère politique du théâtre arabe (j'entends ici par « théâtre » à la fois les formes du théâtre incarné existant en Orient depuis le $\mathrm{XIX}^{\mathrm{e}}$ siècle, mais également toutes les formes de « proto-théâtre » et de représentation existant dans la culture traditionnelle arabe depuis l'ante-Islam) n'est pas un phénomène nouveau, il serait même, comme le souligne Ma'sud Hamdan dans son ouvrage Poetics, politics and protest in the Arabic theatre ${ }^{3}$, un élément constitutif de celui-ci, y compris à travers la dimension satirique des farces et récits arabes traditionnels.

Or, ce qui attire tout particulièrement notre attention ici, est que ce théâtre adopte pour ce faire des esthétiques propres à la tradition du théâtre politique en Occident, que ce soit le théâtre documentaire chez Soliman, ou le théâtre épique chez el-Attar. Ce phénomène n'est pas nouveau et a des précédents historiques. Dans les années 1950, au moment où l'Égypte connaît un renouveau théâtral au début de la présidence de Nasser, le dramaturge allemand Bertolt Brecht devient une référence «tant pour ses positions théoriques qu'idéologiques ${ }^{4} \gg$. D’après M. M. Badawi, les auteurs égyptiens, 
pour défendre les théories nouvelles du socialisme arabe, sont influencés par les théories occidentales du «théâtre global » et de la distanciation ${ }^{5}$. C'est donc des esthétiques quasiment canoniques du théâtre politique occidental que s'approprient les artistes pour aborder des problématiques proprement arabes. Ce renouveau théâtral est dans un premier temps encouragé par le régime et les institutions. Pourtant, ce qui se joue là est l'intégration par Gamal Abdel Nasser du théâtre à un réseau de médias au service de la propagande. Dès les années 1960, la censure augmente, et les dramaturges n'expriment plus leurs idées que de manière détournée. Sous la présidence d'Anouar el-Sadate, les politiques de censure restreignent les thèmes abordés dans les pièces, et se doublent d'une politique économique d'ouverture au libéralisme (Infitah). Le théâtre se vide en grande partie de sa substance politique, et entre en concurrence directe avec les mass médias. C'est la fin d'une période de soutien de l'État aux innovations esthétiques au théâtre.

Dans ce contexte apparait à la fin des années 1980 une génération d'artistes indépendants à laquelle appartient Ahmed el-Attar, qui a fondé sa compagnie el-Ma`bad («Le Temple ») au début des années 1990, et à laquelle viendra s'agréger Laila Soliman dans les années 2000. Ces artistes ont en commun d'avoir voyagé ou vécu en Occident, ou encore d'avoir été élevé dans une double culture. Ils cherchent à renouveler la scène égyptienne, notamment en abordant sur la scène des questions sociales, politiques, économiques, et en proposant un point de vue différent de celui affirmé par les médias nationaux, les discours politiques ou le système éducatif public. Ils importent d'Occident une culture théâtrale qu' ils redécouvrent à laquelle ils empruntent des esthétiques. C'est ainsi par une nouvelle rencontre avec l'Occident, dans un contexte certes très différent de celui des années 1950, qu'apparaît un nouveau théâtre expérimental en Égypte.

Le dramaturge et metteur en scène Ahmed el-Attar fonde sa compagnie, el-Ma'bad («Le Temple ») en 1992, après avoir obtenu un diplôme en Études théâtrales à l'Université américaine du Caire. Parfaitement francophone, il a vécu huit ans en France. Au début des années 2000, il complète sa formation par l'obtention d'un master en Management culturel à la Sorbonne. S'il connaît sans aucun doute les grands classiques de la littérature orientale, il reconnaît volontiers que sa culture théâtrale est internationale : «Une part importante de mon apprentissage s'est faite en regardant des pièces venues du monde entier - cela a, de manières multiples, formé mon 
regard $^{6} . \gg$ Il dénonce par ailleurs l'absence de cette culture théâtrale internationale en Égypte, à laquelle il tente de pallier en invitant à la fois des artistes égyptiens et étrangers au Dowtown Cairo Art Festival, qu'il organise annuellement depuis 2012.

Depuis les années 1990, deux grands thèmes semblent sous-tendent son œuvre: la vie quotidienne sous le régime militaire autoritaire et la manière dont cela transforme les relations entre les individus d'une part, et le rapport des Égyptiens à l'Occident et à ses mythes d'autre part. Nous nous intéresserons tout particulièrement ici à une création de 2004, Mother I Want to Be a Millionnaire. Cette pièce interroge le rapport des Égyptiens à l'argent et à un mode de vie occidental fantasmé, questionnant ainsi leur rapport biaisé à l'existence quotidienne. Ce faisant, c'est aussi la frontière entre illusion ou plutôt, désillusion dont sont victimes les personnages - et réalité qui est explorée. La pièce raconte l'histoire d'un jeune homme, Hassan, sans emploi comme une grande majorité des jeunes égyptiens, qui participe à une émission de téléréalité diffusée à l'échelle du monde arabe, dans laquelle les participants doivent à la fois chanter et convaincre des téléspectateurs fictifs de voter pour eux, face caméra. La pièce questionne le rapport des Égyptiens aux médias de masse, à la célébrité et à l'argent, mais au-delà, fait la critique d'une jeunesse sans avenir et sans espoir qui s'engouffre dans les brèches creusées par le néo-libéralisme et l'influence américaine, en rupture avec les modes d'échanges traditionnels des sociétés orientales. Il expose le fossé existant entre le conservatisme de la société égyptienne et les fantasmes véhiculés par les médias.

Du point de vue de la dramaturgie et du dispositif scénique, l'héritage du théâtre épique brechtien est évident. La narration n'est pas linéaire. La pièce est découpée en 51 tableaux, en fait huit séquences qui reviennent de manière aléatoire et proposent chacune un point de vue différent sur la situation. Cette dramaturgie repose donc sur une esthétique du montage, qui est l'une des dix-neuf caractéristiques du théâtre épique chez Brecht, tel qu'il le définit dans son essai «Sur une dramaturgie non aristotélicienne ${ }^{7}$ » et qui consiste, selon la définition proposée par Jean-Pierre Morel en «l'agencement, dans un certain ordre, d'éléments séparés, relativement autonomes, au service d'une fin déterminée ${ }^{8} \gg$. Ainsi, dans la pièce, les tableaux de la participation des candidats à l'émission de télé-réalité sont entrecoupés par le récit des aventures de «Hassan », héros d'une fable populaire égyptienne, d'origine palestinienne ${ }^{9}$, racontée par une femme dont le visage est projeté sur un écran. Ce conte fonctionne comme un récit parabolique de l'aventure du héros de la pièce. Jean-Pierre Morel évoque également, dans le système 
du montage le fait $\mathrm{d}^{\prime} \ll$ intercaler dans le récit de façon ponctuelle $[\ldots]$ des éléments à première vue étrangers au cours des évènements rapportés ${ }^{10} \gg$. La pièce nous en donne un exemple intéressant, lorsque des séquences quasi oniriques laissent apparaître à la scène les employés de la bureaucratie égyptienne, effectuant des actions administratives, comme autant de gestus brechtiens, de manière mécanique. A priori, ces séquences sont étrangères à la fiction, mais la complexité et l'absurdité du système bureaucratique peuvent expliquer l'immobilisme de la société égyptienne et le chômage du jeune Hassan. Enfin, la situation proposée, celle de la participation de Hassan à un télé-crochet, permet tout naturellement à el-Attar d'intégrer au cœur de la dramaturgie des passages chantés par les candidats, et qui fonctionnent comme les songs brechtiens, que le dramaturge et théoricien allemand définit comme des « sortes de réflexions et de commentaires moralisants ${ }^{11} \gg$, et permettent au spectateur de prendre de la distance par rapport à l'action.

Il s'agit alors de s'interroger sur le sens de ces emprunts. La dramaturgie brechtienne a pour objectif une action pédagogique sur le spectateur, l'invitant à se questionner sur sa propre place dans la société et à agir sur le réel. Cette dimension didactique du théâtre n'est peut-être pas absente chez el-Attar, où il pourrait venir pallier les faiblesses du système éducatif égyptien, entretenant les élèves dans l'inculture. Cette critique est directement présente dans le spectacle lors d'une série de tableaux représentant le jeune Hassan à l'école, où l'instituteur ignorant se contente de véhiculer des discours convenus sur la puissance de l'agriculture égyptienne, et où les cours de littérature sont remplacés par des cours de religion. Pourtant, il semblerait ici que le dramaturge cherche davantage à faire réfléchir le spectateur sur l'aliénation du sujet que de l'engager à agir sur le cours d'un réel sur lequel il n'a plus prise. Il s'agit bien ici de faire de l'Homme un « objet d'enquête ${ }^{12}$ » comme le dit Brecht, pour réfléchir à la manière dont les mécanismes socio-économiques le transforment. Ici, Hassan n'est pas un personnage auquel le spectateur est invité à s'identifier, mais le symbole d'une génération égyptienne dont la situation et les comportements sont à analyser. Dans certaines scènes, il est seul, exprime face public son sentiment ( «J'ai toujours l'impression d'être un échec ${ }^{13}$. ), mais il est aussi un candidat parmi d'autres à l'émission, et tous expriment, face à une caméra dont les images sont reproduites sur écran à la scène, leur désir de gloire et d'argent. Tour à tour personnage de conte, personnage monologuant, candidat à une émission de télé réalité, le personnage est fragmenté au même titre que la dramaturgie et devient de manière métonymique une image de la fragmentation du monde. 
L'adaptation de l'esthétique brechtienne au contexte égyptien permet donc de convoquer la capacité critique du spectateur d'une manière nouvelle, en revalorisant son rôle dans l'élaboration du sens, qui permettrait « Un théâtre politique, $[\ldots]$ ou plutôt une pensée politique du théâtre qui reposerait sur une pédagogie de la perception ${ }^{14}$. »

L'esthétique du montage est également au cœur des pièces documentaires de la dramaturge et metteuse en scène Laila Soliman. Le théâtre documentaire, qui s'est développé en Occident dans les années 1960, et fût théorisé par le dramaturge allemand Peter Weiss en 1968, est un héritier des théâtres politiques de Brecht et de Piscator, tant par la visée politique qu' il revendique que dans les esthétiques qu'il adopte. C'est un théâtre qui revendique son ancrage dans le réel en refusant la fiction, et a pour ambition de traiter de sujets liés à l'actualité immédiate, ou, comme le définit Weiss, « une forme de théâtre dont l'objet exclusif est la documentation sur un sujet ${ }^{15} \gg$.

Contrairement à l'épique brechtien, le théâtre documentaire ne semble pas avoir directement influencé les dramaturgies orientales. Cela s'explique probablement par des raisons historiques : le théâtre documentaire, dont les objectifs selon Weiss seraient « la critique du camouflage ${ }^{16}$ » et la « critique de la falsification de la réalité » apparaît en Europe au moment où l'Égypte entre dans une phase de durcissement du régime politique et du renforcement de la propagande étatique, ajoutés à un régime de censure puissant. Pourtant, c'est bien aussi parce qu'il s'affirme comme un moyen de lutter « contre les moyens de communication de masse ${ }^{17} \gg$ que son appropriation par l'artiste égyptienne Laila Soliman semble sinon logique, du moins justifiée.

Née en 1981, Laila Soliman, après avoir étudié à l'école allemande du Caire, a obtenu un diplôme en Études théâtrales et Littérature arabe à l'Université américaine du Caire. Elle a ensuite complété sa formation à Amsterdam, aux Pays-Bas. Elle vit et travaille aujourd'hui entre l'Égypte et la Belgique. Elle monte sa première pièce en 2004. Elle adapte ensuite plusieurs auteurs européens, et parmi eux Harold Pinter et Frank Wedekind. En 2009, elle travaille avec le collectif allemand Rimini Protokoll, qui revendique un travail théâtral entre réalité et fiction, et réalise ses spectacles à partir de la récolte de documents et d'entretiens. Ils réalisent ensemble le spectacle Radio Muezzin, convoquant à la scène cinq muezzins du Caire exposant la réalité de leur métier et décrivant leur quotidien.

Cette expérience marque un tournant dans l'évolution de l'esthétique théâtrale de Soliman. Elle se tourne alors vers la méthode documentaire pour 
construire ses spectacles. Peu de temps après, c'est ainsi par le biais du théâtre documentaire qu'elle aborde les problématiques de la révolution de 2011, notamment dans son spectacle No Time for Art, créé en 2012. Le recours à l'esthétique du théâtre documentaire n'est pas ici le résultat d'un choix a priori, mais s'est imposé à l'artiste au fil des évènements révolutionnaires, alors que Soliman remettait en cause la nécessité-même de son travail dans une période troublée. Elle explique ainsi : «J'ai senti qu'à ce moment je commençais à remettre en question mon propre rôle et à douter de mon utilité. [...] Tout aurait pu être mieux si j'avais été une vidéaste ou une journaliste ${ }^{18}$. $\gg$ Plutôt que l'utilité du théâtre, c'est bien son rapport problématique à la fiction et au réel qu'elle met en question ici, et cette réticence est visible dans le titre même de sa pièce. Mais elle interroge aussi le rôle social de l'artiste dans une société en plein bouleversement.

Le spectacle No Time for Art est construit en quatre parties autonomes, pouvant être jouées à la suite ou séparément. Nous étudierons particulièrement ici la seconde partie, intitulée «NTFA \# 1 ». Laila Soliman a voulu y faire entendre l'histoire d'un de ses amis, Aly Sobhi, comédien et militant, arrêté pendant les manifestations de 2011 et victime de violences policières. Mais, par le biais de ce récit, c'est tout le système de la violence d'État banalisée, masquée par des discours médiatiques falsifiés, qu'elle tend à dénoncer. Le théâtre documentaire de Laila Soliman intègre le témoignage individuel des acteurs de la révolution comme document à part entière, ce qui amène la chercheuse américaine Brinda J. Mehta à le considérer davantage comme du « théâtre-témoignage ${ }^{19}$ », sauf à considérer le témoin lui-même comme document vivant. Les témoins sont le plus souvent présents en scène, comme c'est le cas d'Aly Sobhi, qui raconte lui-même au public son arrestation. Ce témoignage est alors confronté au récit officiel de l'arrestation des « voyous » de la place Tahrir, diffusé par la télévision nationale. Sobhi apparaît sur les images diffusées par les médias, parmi une foule de jeunes gens désignés par le commentateur comme « des perturbateurs et des voyous » arrêtés place Tahrir. Devant le groupe de détenus sont disposées des armes de toutes sortes, désignées par le commentateur comme «des armes blanches, des quantités de cocktails Molotov ainsi que des tubes à gaz, devant servir à susciter le chaos et déclencher des actes hostiles qui menacent à la fois la sécurité nationale et celle des citoyens ${ }^{20} \gg$. Le témoignage de Sobhi à la scène vient alors dénoncer la falsification de la vérité par les médias, en racontant que ces images ont été mises en scène par les forces armées pour la télévision : « Il y avait un officier ensemblier qui préparait le plateau, aidé de deux assistants 
[...]. Les soldats ont apporté les couteaux, les Molotov et les bombes de thé et ils les ont étalés sur la table. »

Laila Soliman confronte ici des documents, les manipule, les organise afin de structurer le réel, et de mettre en lumière des pans de la réalité falsifiée mise en place par les institutions. Elle propose donc au spectateur une nouvelle lecture des informations qui lui dont présentées au quotidien, pour appeler à un regard critique.

Ces deux artistes ont donc pour ambition de questionner par leur théâtre la société dans laquelle ils vivent, en adoptant un point de vue souvent très critique par rapport aux politiques nationales, ce qui nous amène à interroger leur liberté de création, dans un régime où la censure est très forte. Ces artistes

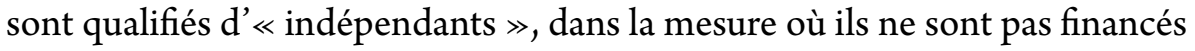
par les institutions publiques. Cette position leur permettrait alors d'éviter en partie les politiques de censure, afin d'aborder des problématiques sociales et politiques, reconnectant ainsi le théâtre à la sphère civique. El-Attar explique en partie cela par l'esthétique d'avant-garde de ses pièces, qui en fait des objets de fait réservés à une élite intellectuelle, et par la taille réduite des lieux de représentation, et partant, du nombre de spectateurs :

[Sous Moubarak], la censure n'était pas toujours appliquée. Au contraire, le régime s'intéressait surtout aux médias de masse. Il ne prêtait pas attention à 2000 ou 5000 personnes qui pouvaient assister à telle pièce spécifique programmée pendant cinq semaines. Et cela est logique, ces nombres sont inférieurs à la population d'une seule rue du centre-ville du Caire. [... ] Tu faisais ta pièce, tes amis, la vieille élite intellectuelle, et peut-être quelques Égyptiens âgés viendraient la voir. Les autorités n'en ont tout simplement rien à faire de toi ${ }^{21}$.

Ainsi, la liberté relative de ce théâtre expérimental indépendant aurait pour conséquences des moyens financiers limités et un public restreint. Cette double contrainte a alors entraîné les artistes à nouer de nouveaux liens, institutionnels cette fois-ci, avec l'Occident, et avec l'Europe en particulier, à la fois pour trouver des financements et une audience plus large en présentant leurs spectacles sur les scènes européennes.

Il faut donc considérer ici le lien entre les artistes égyptiens et l'Occident à la fois d'un point de vue artistique et institutionnel. Le lien apparaît comme fécond, permettant le renouvellement des formes artistiques et l'ouverture à un nouvel horizon de création, mais nous invite également à nous interroger sur le rapport des artistes aux destinataires de leurs créations - cela est d'autant plus vrai dans le cas d'un théâtre politique, comme le souligne $\mathrm{O}$. 
Neveux dans son ouvrage Politiques du spectateur ${ }^{22}$. À qui s'adressent ces créations? Si ces spectacles s'adressent à un public égyptien mais sont joués à l'étranger, faire un théâtre politique en dehors de la cité est-il possible? Si ces spectacles sont pensés pour un spectateur européen, comment faire en sorte que l'ancrage égyptien ne se résume pas à un simple folklore ou ne soit pas irrecevable à cause de barrières langagières ou culturelles? Il s'agit donc enfin de nous demander comment ces artistes parviennent à construire des objets transculturels qui ont du sens dans un double contexte, en envisageant une diffusion des spectacles à une nouvelle échelle.

Le spectacle On the importance of being an Arab, réalisé par Ahmed el-Attar en 2009, apparaît ainsi comme un objet construit pour un double public, à la fois égyptien et européen, sans pour autant entraîner les mêmes effets dans sa réception par l'un ou l'autre de ces publics. Dans ce spectacle, que el-Attar décrit lui-même comme « une forme hybride entre l'installation et la performance $^{23} \gg$, le dramaturge propose un dispositif de monologue, et prend luimême la parole seul en scène, face public. Le texte est composé d'un montage de ses propres conversations téléphoniques, enregistrées pendant deux mois. Il n'est jamais donné au spectateur d'entendre l'interlocuteur, dont on devine cependant parfois l'identité. Ce dispositif est complété par une musique lancinante ininterrompue, et par la projection, sur un écran en fond de scène, d'extraits de journaux intimes et de lettres écrites par el-Attar lui-même, comme autant de documents nous renseignant sur la vie de l'auteur. El-Attar revendique l'usage de ce matériau très personnel qui a servi à la création du spectacle : «En fait, le spectacle est à la fois une synthèse visuelle, sonore et dramaturgique de la vie d'un Égyptien dans l'Égypte d'aujourd'hui. Et cet Égyptien, c'est moi ${ }^{24}$. »

Les conversations téléphoniques abordent les thèmes de la visite de Barack Obama au Caire, en 2009, dénonçant l'apparat politique en opposition à la réalité sociale de la misère en Égypte, questionnent le statut de l'artiste dans la société égyptienne, et mettent en lumière l'absurdité du système bureaucratique dans le pays. Mais Ahmed el-Attar nous laisse aussi entendre des conversations abordant des thèmes plus intimes, celui de son mariage ou celui du conflit avec son père. C'est donc un « je » autobiographique, qui vient présenter sa position dans l'espace social, et expose, par le contenu de ses propos, sa conscience politique. Mais c'est aussi un « moi $\gg$ intime qui se présente au spectateur, où les éléments politiques vécus collectivement rencontrent des moments de vie intime. Le locuteur assume ici une position conflictuelle par 
rapport à la société dans laquelle il vit. La parole individuelle émerge donc ici pour dépasser une désagrégation du moi et redonner une place à l'individu au sein d'une société qui tend à uniformiser. Par le monologue, l'auteur-acteur trouve une liberté d'expression, et confronte les spectateurs à une réalité qui est également la leur. Ils sont convoqués, par la frontalité du dispositif, à remettre en cause la société. De la même manière que la présence scénique n'est plus celle du personnage mais celle de l'auteur comme citoyen agissant dans le monde, la communauté des spectateurs cesse d'être fictive. Le spectateur égyptien est amené à voir ici une image de ce qu'il est, autre que celle construite par un double discours, le discours national égyptien d'une part, et le discours occidental d'autre part. Pour un public égyptien, le spectacle possède alors une dimension militante. Mais ce spectacle s'adresse également à un public occidental. Dès le titre, que l'on pourrait qualifier de provocateur, le spectacle invite à réenvisager la manière dont vit un Arabe aujourd'hui, en se dégageant des stéréotypes. Il opère comme une invitation à découvrir l'Autre comme altérité (au sens d'Emmanuel Levinas), afin de déconstruire une représentation de l'Arabe telle qu'elle a été construite depuis le $\mathrm{XIX}^{\mathrm{e}}$ siècle par l'Occident, comme le démontre Timothy Mitchell dans son essai Colonising Egypt ${ }^{25}$.

C'est un autre contact entre Orient et Occident que propose Laila Soliman dans son dernier spectacle, Zig Zig (2016), où elle traite d'un évènement commun à l'histoire anglaise et à l'histoire égyptienne. Dans son précédent spectacle, Hawa el-horreya, présenté à Londres, elle interrogeait les conséquences de la Seconde guerre mondiale sur les politiques coloniales de la Grande-Bretagne en Égypte, et la manière dont l'affaiblissement de la puissance occidentale avait permis l'émergence du mouvement révolutionnaire de 1919 contre l'occupation anglaise. Dans Zig Zig, elle aborde un autre épisode méconnu de l'occupation: les viols et les pillages commis par les soldats anglais dans le village de Nazlat el-Shobak, près de Giza. Les témoignages de ces victimes avaient ensuite nourri les pamphlets des nationalistes contre les occupants, et les exactions avaient fait l'objet d'un procès, demeuré sans suite. Le texte du spectacle, conçu à partir des documents d'archives sur le procès trouvés au British Foreign Office, est porté à la scène par quatre jeunes comédiennes et une musicienne.

Contrairement à ce que l'on pourrait penser, ce spectacle n'est pas un spectacle à charge contre les Anglais. Si les documents mettent en lumière la nature violente de l'occupation, c'est avant tout le rapport des femmes violées à Nazlat el-Shobak à la violence qui est interrogé. Les comédiennes se dé- 
tachent ainsi par moment de la documentation pour évoquer leur rapport personnel à la violence généralisée, et notamment celle à l'œuvre aujourd'hui dans la société égyptienne, question réactivée par les multiples agressions de femmes lors des manifestations de la place Tahrir. Le spectacle met ainsi en place un lien entre passé et présent, entre la violence coloniale et la violence quotidienne en Égypte, et la manière dont cette violence est tour à tour tue et instrumentalisée, sans que les femmes, premières victimes, ne soient replacées au centre des débats.

Ces deux spectacles témoignent de la vitalité du contact entre Orient et Occident, afin de porter à la scène et d'explorer des problématiques communes, d'enrichir la réflexion sur un sujet de société ou de chercher à remédier à l'idée d'un « choc des civilisations », concept théorisé par Samuel Huntington en 1996, depuis largement critiqué mais qui tend à refaire surface.

Depuis les années 1990, l'influence des esthétiques théâtrales occidentale a permis à une jeune génération d'artistes égyptiens d'enrichir leur travail et de réenvisager la place du théâtre dans la société, mais aussi de présenter leurs créations à une échelle internationale. Ce mouvement est allé en s'accentuant, et a trouvé un nouveau souffle avec la Révolution de 2011, qui a entraîné le public occidental et les programmateurs de théâtre à jeter un regard nouveau sur la jeune génération arabe. Cela est notamment remarquable dans la programmation du Festival d'Avignon, qui a intégré en 2014 un spectacle de la compagnie égyptienne El-Warsha, puis en 2015 la pièce The Last Supper d'Ahmed el-Attar, et qui a présenté cette année la dernière pièce du metteur en scène syrien Omar Abu Saada. Cet essor de la programmation de spectacles arabes dans une grande institution est ainsi symptomatique, même s'il ne faut pas oublier que certaines salles et festivals ont intégré les dramaturgies arabes à leurs programmations de manière plus régulière et depuis plus longtemps (c'est le cas du Tarmac, ou de Confluences, à Paris, ou des Rencontres à l'échelle qui ont lieu chaque année à Marseille).

Dans cette perspective, les artistes arabes ont dû s'adapter à ce nouveau public, de manière plus ou moins rapide et plus ou moins régulière. Néanmoins, la gageure a été de proposer des objets artistiques à la fois recevables par un public étranger, mais conservant du sens pour le public local.

Cette adaptation des dramaturgies arabes semble nécessaire, afin que le lien entre Orient et Occident ne soit pas réduit à un lien économique et institutionnel, mais permette un renouvellement des enjeux esthétiques et politiques du théâtre. 
Transfert des esthétiques politiques du théâtre européen dans le contexte égyptien...

\section{Bibliographie}

Badawi Muhammad Mustafa, Modern Arabic drama in Egypt, Cambridge, Cambridge University Press, 1987.

Béhague Emmanuel, «Brecht et l'écriture dramatique contemporaine - héritages et confrontations ", in M. Silhouette et J. M. Valentin (dir.), Bertolt Brecht, la théorie dramatique, Klincksieck, 2012.

Brecht Bertolt, Écrits sur le théâtre 1, Paris, L'Arche, 1963.

Fahim Joseph, "Theatre before and after a revolution " [en ligne] in American Theater, May-June 2014, consulté le 04/03/2016, URL: http://www.americantheatre.org/2014/05/10/theatre-in-egypt-before-and-after-the-revolution-2/ view-all/

Hamdan Ma'sud, Poetics, Politics and protest in Arab theatre - The bitter cup and the holy rain, Brighton - Portland, Sussex Academic Press, 2006.

Mehta Brinda J., «The darker side of Tahrir in Laila Soliman's No time for art and Blue Bra day: Scene two - Tahrir Square, Cairo ", in Dissident Writings of Arab Women, Londres - New York, Routledge, 2014.

Mitchell Timothy, "Chapter 1: Egypt at the Exhibition " in Colonising Egypt, Berkeley, University of California Press, 1991.

Morel Jean-Pierre, «Brecht et la question du montage dans les années 30 » in M. Silhouette et J. M. Valentin (dir.), Bertolt Brecht, la théorie dramatique, Klincksieck, 2012.

Neveux Olivier, Politiques du spectateur - Les enjeux du théâtre politique aujourd'hui, Paris, La Découverte, 2013.

Soliman, Laila, conférence "Truth is concrete ", Bruxelles, 24 septembre 2012 [Vidéo disponible en ligne], consultée le 03/03/2013, URL: https://www. youtube.com/watch?v=mfDvJsBhtZw/

Weiss Peter, « Notes sur le théâtre documentaire » in Discours sur la genèse et le dérou-lement de la très longue guerre de libération du Vietnam illustrant la nécessité de la lutte armée des opprimés contre leurs oppresseurs, traduit de l'allemand par J. Baudrillard, Paris, Éd. du Seuil, 1968.

\section{Notes}

1. Olivier Neveux, Politiques du spectateur - Les enjeux du théâtre politique aujourd' hui, La Découverte, Paris, 2013, p. 80.

2. Ibid., p. 42.

3. Ma'sud Hamdan, Poetics, Politics and protest in Arab theatre - The bitter cup and the holy rain, Sussex Academic Press, Brighton - Portland, 2006.

4. Muhammad Mustafa Badawi, Modern Arabic drama in Egypt, Cambridge, Cambridge University Press, 1987.

5. Ibid., p. 140 . 
6. Propos recueillis par Joseph Fahim, op. cit.

7. Bertolt Brecht, «Sur une dramaturgie non aristotélicienne », in Écrits sur le théâtre 1, L'Arche Éditions, Paris, 1963 (pour la traduction française), p. 260.

8. Jean-Pierre Morel, «Brecht et la question du montage dans les années 30 » in $\mathbf{M}$. Silhouette et J. M. Valentin (dir.), Bertolt Brecht, la théorie dramatique, Klincksieck, 2012, p. 61.

9. Fable connue en Égypte sous le titre de « Shâtir Hassan » ou «Le Malin Hassan ».

10. Ibid., p. 70.

11. Bertolt Brecht, «Sur l'architecture scénique et la musique », in Écrits sur le théâtre 1, op. cit., p. 454.

12. Bertolt Brecht, «Sur une dramaturgie non aristotélicienne », op. cit., p. 261.

13. Extrait du texte Mother, I want to be a millionnaire, Ahmed el-Attar, non publié. Traduction personnelle.

14. Emmanuel Béhague, «Brecht et l'écriture dramatique contemporaine - héritages et confrontations ", in M. Silhouette et J. M. Valentin (dir.), op. cit., p. 159.

15. Peter Weiss, " Notes sur le théâtre documentaire " in Discours sur la genèse et le déroulement de la très longue guerre de libération du Vietnam illustrant la nécessité de la lutte armée des opprimés contre leurs oppresseurs, traduit de l'allemand par J. Baudrillard, Éd. du Seuil, 1968, p. 7.

16. Ibid., p. 8.

17. Ibid., p. 9.

18. Laila Soliman, conférence "Truth is concrete», Bruxelles, 24 septembre 2012. Disponible sur Youtube. Traduction de l'anglais personnelle.

19. Brinda J. Mehta, «The darker side of Tahrir in Laila Soliman's No time for art and Blue Bra day: Scene two - Tahrir Square, Cairo ", in Dissident Writings of Arab Women, Routledge, Londres - New York, 2014, p. 220.

20. Extrait du texte du spectacle No Time for Art, Laila Soliman. Traduction française de Shady el-Hosseiny. Non publié.

21. Propos recueillis par Joseph Fahim, dans l'article «Theatre in Egypt before and after the Revolution », publié sur le site americantheatre.org, mai 2014. Traduction de l'anglais personnelle.

22. Olivier Neveux, Politiques du spectateur - Les enjeux du théâtre politique aujourd'bui, La Découverte, Paris, 2013.

23. Extrait de l'entretien avec Ahmed el-Attar dans le programme du spectacle, présenté au théâtre Le Tarmac, à Paris, en mars 2015.

24. Ibid.

25. Mitchell Timothy, « Chapter 1: Egypt at the Exhibition » in Colonising Egypt, Berkeley, University of California Press, 1991, p. 2-33. 